\title{
Informal caregiving: Cross-cultural applicability of the Person-Environment Model
}

ABSTRACT

KEY WORDS

Sociology, gerontology, aging, caregiving, personenvironment, cross-cultural
The 21st century will be characterised by aged and ageing nations, making eldercare a growing concern. Most eldercare in most nations will be provided informally, primarily by female family members. Helping these people understand the dimensions of eldercare is a key to effective and cost-effective caregiving. The Person-Environment Model (Lawton and Nahemow 1973) is proposed as a theoretical framework for understanding, assessing, and optimising family-based caregiving. This paper presents findings from a qualitative study of informal rehabilitation caregiving provided to elderly stroke survivors in Thailand. Four main rehabilitation dimensions (biological, psychological, social, spiritual) are identified, as are three main caregiver needs (information, assistance, and support). We suggest that while the Person-Environment Model is useful in developed nations, it is perhaps more valuable in societies where fewer options to family-based eldercare exist, and thus where effective informal eldercare is more critical. Implications for education and training of health care providers are also discussed.

\section{Received 24 March $2009 \quad$ Accepted 3 June 2009}

\section{Edwin Rosenberg}

Department of Sociology, Appalachian State University Boone, NC, USA

\section{Pornchai Jullamate}

University of Burapha, Ampur Muang, Chonburi Thailand

\section{Zaida Azeredo}

University of Porto, Porto, Portugal

\section{Introduction}

$\boldsymbol{T}_{\mathrm{cen}}^{\mathrm{t}}$ t seems safe to say that three themes will characterise the first decades of the 21st century: global ageing, the global economic crisis, and continued globalisation in all spheres of life. The sheer demographics of global ageing imply that nearly every nation is or soon will be confronted with the increased pressures of providing economic security and health care for rapidly growing populations of elders. Gerontologists, demographers, economists and other social scientists have been aware of this and have been sounding the warning for decades, often to little avail. The current global economy, while affecting some nations more severely than others, has generally resulted in declining financial resources at a time of rising social need. This makes meeting any demographic challenges more difficult; in particular, programmes and services for elders must vie for funding with programmes and services for non-elders in an increasingly competitive era. Finally, continued globalisation, assisted by newer communication technologies, interconnects nations in more ways than ever before. While this has some negative ramifications (see 'global economic crisis'), it also facilitates international and crosscultural social science inquiry and research. 
One aspect of international/cross-cultural collaboration is the search for 'modified cultural universals': findings, theories, or programmes that have high applicability across many nations/ cultures. In relation to applied gerontology, for instance, which has the overall goal of enhancing the lives of elders, knowledge would be sought that leads to programme development that enriches elders' lives across a variety of nations and/or cultures. While acknowledging the uniqueness of cultures, some commonalities of the human condition are also recognised. It is these commonalities that 'modified cultural universals' uncover and describe. Just as the preferred theory is the most parsimonious one, explaining the most variation with the fewest concepts, the preferred social programme in a global sense is the one that addresses the greatest proportion of needs, multi-nationally, with the fewest variations or adaptations.

The topic of this paper is eldercare. While 'old age' and the indicators of its onset can be defined according to different criteria (e.g. chronological age, functional age), every society has an age group considered 'old', and every society must, one way or another, determine and provide for the welfare of this group. (In some nations with high life expectancy, middle-aged persons will spend more years providing eldercare than they did childcare.) After a discussion of eldercare generally and in Thailand, we will describe the Person-Environment (P-E) Model (Lawton and Nahemow 1973) and test its utility to explain informal caregiving in rural Thailand. We will then discuss the extent to which the P-E Model can be employed cross-culturally to explain the degree of success of various cultures' eldercare structures. In addition to the data from Thailand, this discussion of the need for and structure of eldercare will include data from two other countries with arguably different cultures (e.g. dominant religion) and levels of development, and which are at different stages of the demographic transition from a youthful to an ageing nation: Portugal and the United States. (These represent the authors' home nations. We hope, also, that our different backgrounds sociologist, nurse, medical doctor - will add breadth to our perspective.) Thus the research questions focus on the P-E Model's applicability to rural Thai eldercare in particular and, in general, its application across cultures. Finally, we will discuss the implications of this model for health sciences education, practice, and policy.

\section{Eldercare}

The term 'caregiver' refers to anyone who provides assistance to someone else who is, to some degree, incapacitated and needs help (Family Caregiver Alliance 2003). Eldercare, then, can be viewed as assistance to older persons in performing basic and instrumental activities of daily living.

A distinction should be made between formal eldercare, accessed through private or public programmes and typically provided by directcare workers, and informal eldercare, typically non-paid and provided primarily through family, though also by friends or neighbours (e.g. Emlet et al 2007). Thus informal eldercare refers to various forms of help, including physical, emotional, and financial support, which older persons receive from people they know.

Globally, the vast majority of eldercare is informal. For example, even in the United States, a highly developed nation with a comparatively sophisticated network of residential facilities (e.g. nursing homes) and private/public programmes and services for older adults, and with its stereotype of institutionalising older persons, over $80 \%$ of elders with three or more activities of daily living limitations remain noninstitutionalised due to informal caregiving. Of these community-dwelling elders, only $8 \%$ rely solely on formal care, while about $80 \%$ receive assistance only from informal caregivers, of whom about $80 \%$ are female (Connidis 2001:130; Hooyman and Kiyak 2008:385).

Thus it is not surprising that gerontologists (e.g. Crandall 1980; Hooyman and Kiyak 2008) weight their definitions of eldercare towards its informal dimension, in terms of filial maturity or filial responsibility, i.e. adult children who provide supportive services for their aged parents. Historically such care has been expected, often due to cultural values and 
norms of respect for elders and in reciprocity for the aged parents' earlier care for their children. Ideally the burden is borne willingly. However, modernization (e.g. Cowgill 1974) often creates shifts in family, community and societal roles, thus threatening the ideological and logistical aspects of traditional, informal eldercare.

Many different aspects of the eldercare context can and are evaluated and studied across nations. There are societal costs, such as days absent from work, or reduced hours at work, due to eldercare obligations. There are also societal benefits (the value of unpaid, informal eldercare in the USA is estimated at over $\$$ US300 billion annually [Arno 2006]). On the individual/family level, while eldercare can strengthen intergenerational relationships, the strains of caregiving and the demands on caregivers' time are also substantial: caregiving contains objective and subjective burdens that lead to physical, psycho-emotional, and financial stress. In fact, caregiver stress is a common cause of family-based elder abuse. Because the great majority of those receiving long-term care at home rely exclusively on family members or friends for that care, supporting these caregivers with respite and caregiver education programmes, where available, is essential (Link and Dize 2006). In some nations ageing agencies have Family Caregiver Support specialists or offer formal caregiver effectiveness training programmes (e.g. Jullamate et al 2007; Rosenberg and Gouge 2007). Although most older adults receive care only informally, some are able to supplement family care with services from paid providers (FCA 2003).

\section{Female caregivers}

Around the world, women are much more likely than men to provide eldercare. Women provide the majority of informal eldercare to spouses, parents, parents-in-law, friends and neighbours; in the United States, women comprise more than $80 \%$ of the family caregivers for chronically ill elders (OWL 2003). Wives and daughters are the majority of informal caregivers. Although they are similar in terms of being socialised to care for others, especially family members, they differ greatly in role obligations to their care recipients, and the context in which care is provided ( $\mathrm{Li}$ 2004). For example, older wives typically live with their husbands and are not engaged in paid employment; the same cannot be said for many adult daughters.

The caregiver burden can affect caregiver health. More than one-third of caregivers provide intense and continuing care to others while themselves suffering from poor health (Navaie-Waliser et al 2002). Approximately $25 \%$ of female caregivers have health problems as a result of their caregiving activities (OWL 2003), and are at increased risk of coronary heart disease (Lee et al 2003).

As most caregivers and eldercare providers in the world are women, we should be aware that some caregiving issues are either far more applicable, or even unique, to women.

\section{Eldercare in Thailand}

Thailand, where elders are currently defined as age 60+, is currently experiencing one of the most rapid rates of population ageing in the developing world. (The more recently the demographic transition from a youthful to an ageing nation begins, the faster the rate of change.) According to Thailand's National Statistical Office (1998), the proportion of Thais who are elderly rose from $4.8 \%$ to $6.3 \%$ between 1970 and 1990 . By 1996 older persons comprised $8.4 \%$ of the Thai population (Chayovan and Knodel 1996). Currently $10 \%$ of the population, Thai elderly are projected to reach $13 \%$ of the population in 2025 and $17 \%$ by 2050 (National Statistical Office 1998).

Thailand has done little to prepare itself for this demographic trend in general, in terms of its older population, and in terms of eldercare. For instance, the Thai government seemed slow in recognising the importance of welfare for the elderly until 1992, when it adopted the 1992-2011 National Long-Term Plan of Action for the Elderly. Key measures addressed by the Plan included the dissemination of knowledge to seniors on the need to provide for themselves, on health in general, on disease prevention, and on the importance of proper nutrition and exercise. The Plan also called for the extension of 
social-welfare services for the elderly, particularly for those lacking income or with insufficient income, and for those without familial support. Although Thailand has national policies for the elderly, it still lacks public education mechanisms to prepare people for old age: that is, to ensure Thais are able to grow old gracefully and remain financially secure in their later years.

Given globalisation and Thai modernisation, it is remarkable that Thai society has managed to retain one of the more humane aspects of its culture: the obligation to respect and look after elders. This is due to the traditional view of the Thai hierarchy of social and family positions. Older persons are to be valued, respected, and honoured by younger members of the family and in society. Strictly, seriously, and at every opportunity, Thai culture teaches children to look after older persons. Additionally, Thai people firmly believe in and practise the 'gratitude system' (among many strong doctrines), which obligates them to show gratitude to their parents and, in fact, all elderly (Jullamate 2008).

It logically follows that Thai eldercare is almost entirely informal. Most Thai elders approximately $94 \%$ - share a house with their children and rely on their family for support, regardless of whether or not their children are married and have their own parenting responsibilities. Elderly Thais who do not reside with their children are visited by their children regularly - at least once a month. In both cases, adult children typically provide financial support as well as assistance with basic and instrumental activities of daily living, and psychosocial and spiritual care (Jullamate 2008).

Thus Thai family members - in particular adult children - continue to be the foundation of active, responsible care of their aged parents, and often also for their extended family elders, once the elders are no longer able to fully function on their resources. At this time it is clear that eldercare in Thailand is almost entirely informal, and mostly kin-based. Little formal care - i.e. care provided by the government or for-profit organisations - exists. In Thailand today, in most circumstances, the best care providers for the elderly are family members, whose loving support is vital for the physical, emotional and mental well-being of older people.

\section{The Person-Environment Model}

The Person-Environment (P-E) Model (Lawton and Nahemow 1973) is an important framework for describing and explaining the impact of all aspects of the external environment on older persons. (It has also been used in psychotherapy [Neufeld et al 2006] and studies ranging from occupational stress [Baker 1985] to adolescent antisocial behaviour [Loeber and StouthamerLoeber 1998]). One value of the P-E Model is that, given a description and explanation of the situation in question, it can then help comparatively evaluate proposed interventions, modifications or programmes to enable older persons to better cope within their environments. Another value is that the model can be applied at either the micro level (e.g. modifying the home of an older person who has suffered a stroke so that he/she can continue to live in it) or the macro level (e.g. evaluating the impact of proposed changes in a government's role in elders' financial security or health care options).

Since cultures vary but certain human needs (economic sufficiency, health care, social support) are arguably universal, a model that assists our understanding of and responses to those needs should have cross-cultural and international applicability. The P-E Model, in our opinion, can do just that.

As physiological and psychological entities, people live in and must be able to successfully cope with an environment (home, climate, political/economic situation, etc.) that makes certain demands on them. In the P-E Model, this is referred to as Environmental Press (EP). To meet those demands people use their available resources - e.g. physical, cognitive/emotional, economic, social - that in totality comprise 'Personal Competence' (PC).

Every environment, from micro to macro, exerts some minimal amount of pressure - this is why, in Figure 1, EP does not begin at the zero point (or origin). Whether studying a stroke victim attempting to rehabilitate at home or a society developing long-term care policies (where PC 


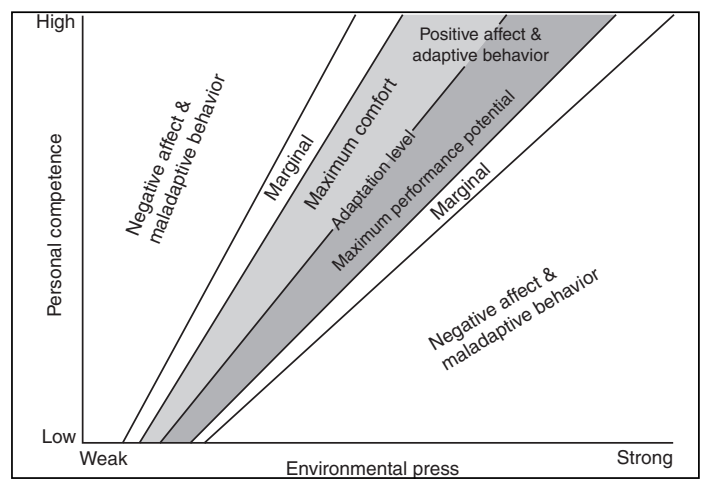

Figure 1 The Person-Environment Model (Lawton and Nahemow 1973).

might become SC: 'societal competence'), EP is assessed or estimated (between the extremes of weak and strong) and, from the EP value, a vertical line is drawn. Following this, PC is assessed or estimated (between the extremes of low and high) and, from the PC value, a horizontal line is drawn. These lines intersect in one of the adaptation level zones.

There are two "positive affect and adaptive behaviour' zones. 'Maximum comfort' is achieved when PC slightly exceeds EP: the total EP is challenging, but the level of $\mathrm{PC}$ leads to confidence that those challenges can be met, albeit with conscious focus and effort. It's like playing a sport or computer/video game you know you can usually win if you make a solid effort.

The 'zone of maximum performance potential' is where EP is roughly equal to PC. In this situation, a person calls upon all their resources/creativity/ingenuity to master the environmental press and, as in sports or games, sometimes a person can surprise themself and exceed their own expectations. Thus, the maximum 'potential' is reached.

There are also zones of 'negative affect and maladaptive behaviour'. If EP greatly exceeds $\mathrm{PC}$, this indicates that a person is unable to cope with the environment. In this case, efforts are unsuccessful, and resources inadequate. The individual copes poorly and becomes unhappy, depressed or angry.

The counter-intuitive zone is where PC greatly exceeds EP: why is that a zone of maladaptive behaviour and negative affect? In this situation, things are too easy. If this were a game, you would know you could win every time without much effort; it would soon become boring. If an elderly woman is in a nursing home when she could cope quite well with home and communitybased services, she is being over-treated; her PC is much greater than the nursing home EP. She does not need to be there, will likely be unhappy with her situation, and her behaviours may reflect that. For older persons, the danger of such environments is that they can create dependency where there was none to begin with.

The marginal zones now make sense: they represent the situations where one is at risk of negative affect/maladaptive behaviour, but not yet there. Appropriate and timely interventions can restore the P-E balance to one of the zones of positive affect and adaptive behaviour.

\section{Methodology}

To test the applicability of the P-EModel to informal eldercare, and to provide a basis for consideration of the P-E Model for cross-cultural eldercare utility, we conducted a qualitative study of caregivers of elderly stroke victims in a rural area of Thailand. Participants came from four parts of Chonburi (in Eastern Thailand), a rural-urban province that contains the region's healthcare centre. The sample was purposive and convenient, comprised of subjects self-identified as informal primary caregivers who had assumed their eldercare role when their stroke-surviving relative was discharged from hospital. These caregivers lived with their dependent stroke relatives and provided care and informal rehabilitation activities at home. They received no financial reimbursement.

The interview guide was developed in English, translated into Thai, and back-translated into English. It was pre-tested on three Thai stroke caregivers, who were not included in this study's database, and revised as needed. The result was a 60-90 minute semi-structured interview, conducted in the home (by this article's second author). Stroke survivors were not present during the interviews, which were audiotaped with the caregiver's permission. Field notes and photographs were taken, also with permission. 
The interviews focussed upon the Thai caregivers' informal rehabilitation activities, their reasons for particular activities, their own needs and emotions, and their perceptions of the roles of health professionals in home-based informal rehabilitation.

Data collection ceased when redundancy of themes occurred and no new information was forthcoming; this was after 20 caregivers had been interviewed. The interview tapes were transcribed, and the transcripts reviewed by participants to validate and verify their responses. Pre-categories had been established based on the researcher's knowledge of the context (Coffey and Atkinson 1996). Final categories were established via content analysis of the interview transcripts. Nonparticipant observation data and photographs were also analysed to add information.

\section{Results}

The 20 stroke survivors were evenly divided between males and females, with a mean age of 73.5 years. Three-quarters of the caregivers were female, with a mean age of 53.1 years. Four of the five male caregivers were husbands of the care recipient; the other was a son. Of the 20 caregivers, 13 were married and 5 were single (one caregiver was widowed and one divorced). Twelve had not completed secondary school. Half said they had to be available to provide care 24 hours per day.

Four major dimensions of informal stroke rehabilitation activities emerged from the content analysis: biological, psychological, social, and spiritual. Biological rehabilitation was the most frequent informal rehabilitation activity reported by the Thai caregivers. The four systems of which the caregivers were constantly mindful were musculoskeletal (17 of 20 respondents), respiratory and circulatory (12 of 20), and nervous (8 of 20). Caregivers also paid close attention to skin integrity and nutritional intake. They used various methods to rehabilitate each system, including massage, exercise, mobilisation, and traditional medicine and herbs.

Caregivers also focussed on psychological rehabilitation of the elderly stroke survivors. They deliberately provided opportunities for the stroke survivors to talk with their neighbours outside the home, avoided talking about unpleasant things or events, and rearranged the home environment to facilitate movement and make it easier for survivors to perform what, prior to their stroke, had been their favourite activities. Caregivers tried to anticipate, frequently asking patients about their needs and wishes and trying to satisfy them insofar as possible. Those who were not totally dependent were taken shopping or visiting. Talking, playing and staying with grandchildren were other common and reportedly meaningful psychological rehabilitation activities provided by the caregivers.

Social rehabilitation was delivered by a variety of activities which could be classified into two subcategories. One subcategory is comprised of activities related to patient-social network interaction; the second involves activities related to the patient's pre-stroke social activities. Caregivers provided their stroke relatives with opportunities to meet and talk with neighbours nearly daily, took them on tours, and asked friends and relatives to visit the survivors at home. Other social activities included taking the survivors shopping, arranging social events (such as birthday parties), and aiding participation in their preferred religious activities. Other pre-stroke social activities had been watching favourite television programmes and listening to radio programmes.

Most Thais are Buddhist, and religious activities were often used to promote psychological and spiritual health. Images of Buddha were placed throughout the home, and local Buddhist monks were invited to visit the patients. The caregivers tried to rehabilitate their stroke relative's spirituality by performing or providing many activities involving or related to religion. Two subcategories - activities related to religion, and activities related to superstition - emerged from the content analysis. Spiritual rehabilitation activities related to religion included donating (things or food) to monks or the poor, helping the patients perform religious activities, praying to religious images or symbols, and having the monk sprinkle holy water on the stroke patient; activities related to superstition included helping 
the patients perform superstitious or ritualistic activities (drinking holy water, bathing with holy water, attaching religious pictures or symbols to the wall), and making talismans or magic objects available for the patients to wear.

In addition to information about stroke rehabilitation activities performed by family caregivers, the interviews revealed perceived needs of the caregivers themselves, which fell into three categories: assistance (with caregiving tasks), information (caregiver education and training), and support (financial, social and spiritual). The most frequently mentioned assistance need was help with physical rehabilitation tasks, followed by assistance with the psychoemotional component of caregiving. These findings are consistent with those of similar studies (Bakas et al 2002; Mak et al 2007).

\section{Discussion}

\section{Informal stroke rehabilitation dimensions}

In analysing the biological rehabilitation observations, it seems that because stroke leaves the survivors with many physical disabilities or handicaps, their caregivers did their best to improve or eliminate residual functional deficits. They firmly desired and fully expected their stroke relatives to recover without impairments and to be able to independently perform their activities of daily living and other important activities as well as they had pre-stroke. Therefore they used a great a variety of methods to rehabilitate biological functions, believing that better health would 'empower' the stroke survivors, with consequent benefits for both the survivor and the caregiver.

Psychological problems are common in stroke victims, particularly elders (Berg et al 2003; Kotila et al 1998; Williams et al 2005). Perceiving psychological health to be a significant component of overall wellbeing, all caregivers tried to maximise the recovery of psychological health for their relatives. In addition, Thai culture mandates that (adult) children care for elders and make them as content as possible, especially when they are sick.

Social participation is often abruptly limited after a stroke (White and Johnstone 2000). Caregivers believed that re-establishing and promoting social interactions would be a source of contentment for the stroke survivors. Interacting with neighbours, relatives and friends, and joining in social activities is very common in rural areas and was viewed as an effective means of resuming social participation. Moreover, due to cultural dictates and the Buddhist gratitude system in Thailand, assisting stroke relatives in their preferred social activities showed the caregivers' gratitude to their stroke relatives.

Spirituality, for many people, is a necessary component of life, and can be related to optimism and positive affect (Chally and Carlson 2004). It is well established that religious beliefs may affect psychological wellbeing and coping abilities of older adults, and may play a positive and effective role in recovering from illness (Mackenzie et al 2000). In this study, both caregivers and stroke survivors were Buddhist, and rehabilitating or enhancing the patient's spiritual health by facilitating religious activities would be consistent with Buddhist teachings.

\section{Caregivers' perceived needs}

Most caregivers expressed a need for more information about stroke and, in particular, stroke rehabilitation activities; they felt their current information was insufficient and perhaps incorrect. This follows Portnow and Houtmann (1987), who argue that with accurate knowledge and an attitude of willingness, nearly anyone can learn to provide even complex and intense eldercare at home, often with better outcomes than institutionalised care would yield. Since many developing nations, for varied reasons, lack extensive institutional care, resources allocated to informal caregiver education and training would seem to be well spent.

The primary support needs were financial: a lack of money, and help managing finances. Incomes are low in this rural area; caregivers' mean monthly income was about 12,500 Thai baht (roughly equivalent to US\$370). Specific costs mentioned were those needed to create a more comfortable home environment for the stroke survivor, to purchase rehabilitation items and other necessary medical equipment, and to transport the stroke survivor (since the hospital was not close by and its home health team rarely visited this community), 
as well as the cost of any additional medical home- or hospital-based medical treatment.

\section{Eldercare in Portugal: A nation in transition}

Life expectancy was around 50 years well into the 20th century in Portugal. Few people reached 'retirement age', most lived in traditional (extended) families, and many elders were functionally illiterate. The family took care of its elders, who were not expected to lead active lives, but who were useful as advisers and respected for their knowledge and experience.

However, after the Carnation Revolution of 1974, family forms became more diverse and less permanent, health and social services improved (since all had access to the National Health Service), leading to gains in life expectancy (currently 78 years [World Life Expectancy Map]), the national economy and standard of living improved, and Portugal began to reap the benefits of emerging technologies, globalisation, and European Union membership. Older people were more likely to reach retirement age and to live longer after retirement, but they were no longer viewed by default as valuable advisers to either their professions or their families.

The Portuguese experience seemed consistent with Modernization Theory (Cowgill 1974). Older persons increasingly were viewed as out-dated and useful only as economic consumers. For many elders, illiteracy and years of inactivity were obstacles to productive roles. Most older women had been mothers and homemakers; if they worked, it was usually in low-level agricultural jobs. Retirement incomes remained low, and elders found themselves devalued members of society.

$\mathrm{By}$ the end of the 20th century, however, the traits and social status of older Portuguese people had begun to improve. The oldest cohort of elders was dying out, replaced by a new, more literate, better educated, more financially secure youngold (age 65-74) generation. This generation felt a need to reassert its relevance to society; the increasing prevalence of grandparents raising grandchildren was one visible indicator that elders still had valued roles to play. For the first time Portuguese elders challenged en masse ageist stigmas and stereotypes.
In Portugal today, age 65 is considered the onset of old age. As in Thailand, the proportion of elderly people in Portugal is growing rapidly: the proportion age $65+(13.6 \%$ in 1991$)$ had risen to $17.3 \%$ by 2006 , and is projected to reach $28.6 \%$ by 2040 (INE 2007). Increased life expectancy, especially in the later years, is usually accompanied by chronic conditions and diseases, which are on the rise in Portugal. These reduce older people's functionality and increase their dependence, putting pressure on their own, their family's, and local/regional/national resources.

In Portugal, the 'traditional' extended family is being replaced by nuclear families, which respond better to a modernising economy that values geographic mobility. Blended and single-parent families are also becoming more common. All these affect the nature of family-based eldercare. How close by are the adult children? What are the eldercare obligations or expectations in blended families? How much parental care can a single mother reasonably be expected to provide? What other informal or formal supports are available for the ageing person/couple?

In the past, and to some extent today, Portuguese health and social services were neither sufficient nor prepared to meet elders' needs. Even with a full effort by the Portuguese government, it will likely take several years to develop the quantity and quality of services necessitated by the rapidly growing older population and the changing family and societal age-related dynamics.

\section{Eldercare in the United States: The formal system falters}

In a rapidly ageing United States, family and friends, rather than public or private service programmes, provide approximately $80 \%$ of eldercare. In 1996, $25 \%$ of the American workforce provided informal care (Dettinger and Clarkberg 2002). About 44.4 million family caregivers contribute 29 billion caregiving hours annually, an average of over 650 hours per person worth over US $\$ 275$ billion at market value. These caregivers assist adult relatives and friends with basic daily activities such as meal preparation, bathing and household chores (Link and Dize 2006). 
In addition to its physical and psychological demands, informal eldercare comes with significant economic costs. For instance, the cost to United States businesses to replace women who quit their jobs because of their caregiving responsibilities has been estimated at US $\$ 3.3$ billion annually. Absenteeism among female employees due to caregiving responsibilities costs businesses almost US $\$ 270$ million per year. The annual cost to businesses because of partial absenteeism (extended lunch breaks, leaving work early or arriving late) due to women's caregiving has been estimated at US $\$ 327$ million. Caregiving-related workday interruptions add another US $\$ 3.8$ billion per year to the burden borne by businesses (FCA 2003).

The financial cost of eldercare fails to spare the caregiver him/herself. The average lifetime loss in total wealth experienced by caregivers due to eldercare is estimated to be US\$659,139 over the lifetime (MetLife 1999). A 1998 study found that $49 \%$ of Baby Boomer female caregivers suffered 'financial hardship' as a result of their caregiving (National Alliance for Caregiving 1998).

Caregivers living in rural communities face different challenges compared to their urban counterparts, including fewer formal services, a lack of medical specialists, transportation difficulties, winter weather problems, greater distances to services, and social isolation. Rural health care experts agree that rural Americans have higher morbidity due to lower income and narrower health insurance coverage (FCA 2009). About $50 \%$ of Americans live outside principal cities, and $20 \%$ live in non-metropolitan areas (AOA 2000).

In the 1930s, the time of the Great Depression, the United States entered what has been called the 'Era of Compassionate Ageism'. For the next half-century (especially the 1930s, 1960s and 1970s), the US government created laws, policies and programmes to 'cure' what it believed were older people's 'problems'. The most substantial results were to greatly reduce elderly poverty and increase access to health care. With few exceptions, beginning with the Reagan administration and continuing to the present,
America has changed course. Whether due to changes in the party in power, and thus political ideology, or due to a weakening economy, the number and funding of programmes and services for older Americans have either decreased or increased at a slower rate.

Americans, perhaps more than Portuguese or Thais, had become accustomed to government provision of needed services. Regarding eldercare, since the 1980s more and more of the burden has shifted from the government to communities and, specifically, families. As the American older population swells and the economy falters, even more of the eldercare burden will fall upon already stressed and financially stretched families. In rural America, with a higher cost per unit of service than in more densely populated urban areas, supporting family caregivers will be more critical than ever.

\section{Applying the Person-Environment Model}

Most older people prefer to live at home as long as possible (Hooyman and Kiyak 2008:435436). A stroke often comes with physical and/ or cognitive consequences that make living at home more challenging. Can the P-E Model help informal caregivers and healthcare providers better understand the dynamic between the stroke victim and his/her environment in order to realistically and optimally rehabilitate personal competence and adapt the environment to restore P-E congruency? This is an especially salient concern in rural areas, where the cost per unit of delivering healthcare or services is higher than in urban areas, and where a tradition of family-based eldercare is matched with a relatively under-developed formal eldercare system.

For the older, home-rehabilitating Thai stroke victim and his/her caregiver, the P-E model begins with the premise that the home environment makes certain demands (exerts a certain 'press' - EP) on the person. The press has multiple dimensions: physical (e.g. activities of daily living), psychological/cognitive (e.g. psycho-emotionally adapting to one's new status as a stroke survivor), financial (e.g. food, transportation, home modifications, health care), as well as the social and spiritual dimensions 
described by the caregivers. The stroke survivor and caregiver try to meet/overcome those demands with the totality of resources that can be brought to bear - that is, personal competence (PC). If $\mathrm{PC}$ is greater than $\mathrm{EP}$, the person is likely to function well enough to remain at home without undue personal or caregiver stress; if $\mathrm{EP}$ is greater than $\mathrm{PC}$, adequate functioning is more problematic, since the person lacks the resources to cope with the environment. Either PC must be improved, or EP must be lessened.

We found that Thai caregivers played an important role in re-establishing P-E congruence. Many informal rehabilitation activities (physical, psychological, social and spiritual) were implemented by informal caregivers to restore as much stroke survivor PC as possible. The caregivers also tried several ways to reduce $\mathrm{EP}$, such as home modifications, providing assistive equipment to help the stroke survivors perform basic and instrumental ADLs, and reducing economic and social challenges with financial and social support and networking.

Assuming people prefer living at home to living in a long-term care institution (if those are even available), people will maximise their use of resources $(\mathrm{PC})$ toward that end. However, if PC will never again rise, the only remaining way to restore $\mathrm{P}$-E balance is to lower the $\mathrm{EP}$ (to where the $\mathrm{EP}$ and $\mathrm{PC}$ lines' intersection is once again in the 'positive' zone; see Figure 1). Still, there may come a time when the remaining $\mathrm{PC}$ is insufficient to meet the home's EP; that is when a person must relocate to a less demanding environment, such as a residential long-term care facility. However, this is rare and difficult for Thai caregivers and survivors since: 1) there are few such facilities or formal care options for stroke survivors; and 2) the Thai bunkhun ('gratitude') system expects Thais to take care of their elderly parents at home, and views moving them to institutions quite negatively.

The P-E Model shows how people use their available resources to deal with the pressures of their environments. Resources can increase or decrease with age; some aspects of one's environment are amenable to adaptation, but others may not be. Sometimes elders find, or can be helped to find, resources they never knew they had - more stable ways to walk or climb stairs, cognitive tricks to minimise forgetfulness, reliance on family and friends. Other times elders environments must be modified, if possible, to bring them into balance with reduced PC.

In Thailand, informal rehabilitation, when taught by health professionals and mastered by caregivers, is an important way caregivers can re-balance PC and EP. We thus recommend that health professionals keep a P-E perspective in mind whenever they teach and give information about informal rehabilitation to caregivers and stroke survivors. Rehabilitation knowledge and activities must contribute to a harmonious balance between PC and EP, relieving factors/conditions of maladaptation. Health professionals must also learn and instruct caregivers about EP-lowering interventions that can help restore P-E balance.

While the P-E Model seems clearly applicable to and beneficial for individuals and families, it can also be applied in a larger, programmes-and-policies sense to help evaluate new local, regional and national initiatives before they are implemented, and as part of the evaluation mechanism for extant policies and programmes. Furthermore, there is obviously interaction between micro and macro ways to affect environmental press and personal competence: in fact, that is the raison d'etre of many support programmes for elders.

Finally, the P-E Model should be amenable to eldercare situations cross-culturally. Regardless of nation or culture, we are dealing with aging homo sapiens in a certain living arrangement. In any sociocultural setting, then, if the critical elements of personal competence and environmental press can be delineated and measured, the level of P-E congruence can be determined and, if the fit is not good, alternatives for adaptation can be comparatively evaluated. This seems true regardless of whether the environment is the home, community or society, and regardless of culture or level of development/modernisation. In this sense the P-E Model is culture-neutral, and therein lies much of its value.

\section{Conclusions}

Nearly all nations are increasingly affected by globalisation, population ageing, and the current 
economic downturn. Most nations, now or in the immediate future, are having to deal with issues of ageing - financial security, health care, social roles - in a time of unforeseen budget shortfalls. One aspect and enhancer of globalisation is the development and dissemination of communication technologies, facilitating international and cross-cultural interaction, inquiry and research. This creates the potential for more effectively understanding social issues and for more effectively weighing and evaluating a wide range of responses, since we can easily examine and comparatively assess options across borders. Despite myriad ways in which each culture is distinct from others, there are also areas of crosscultural overlap and, while fewer still, common threads and themes to the human condition.

One common thread - a 'modified cultural universal', if you will - is the need to provide care for older persons as, with age, illness and disease, they lose functional ability and autonomy. This entails a socio-political response to a biomedical issue, and nations will respond differently due to such factors as political ideology and the level of development of medical science and technology. Nonetheless, the number of persons requiring eldercare will only increase, and flagging national economies, along with other factors, are forcing more and more of the eldercare burden onto the informal care system, whose primary care provider is the family.

Most eldercare is informal and provided at home, even in highly-developed nations with an image of institutionalising ill and lower-functioning elders (e.g. the United States). Moreover, since most eldercare around the world is provided by women, a focus on their needs and concerns is merited. Continuation of life-long patterns promotes successful ageing and adaptation in later life (Baltes and Lang 1997). Thus it can be argued that, if at all possible, elders should age in place (in their own homes vs. in an institution), since this is related to higher levels of happiness and independence (Neal 2007; Pynoos et al 2003).

The focus of this paper is population ageing and associated health care needs, particularly in rural areas, where services are typically underdeveloped and the cost per unit of service delivery is high. Using family-based rehabilitation for older stroke victims in rural Thailand as an illustrative example, common eldercare needs can be identified as assistance, information, and support.

Eldercare occurs within an environment with both macro (societal, cultural) and micro (community, home) dimensions. The PersonEnvironment (P-E) Model, developed by Lawton and Nahemow (1973), is a framework for understanding and explaining the level of success of eldercare, on both macro and micro levels, in terms of the ability of available resources to meet the challenges of the environment (used in the broadest possible sense). We believe this model informs the findings of this study, suggesting that activities, autonomy and quality of life can be understood in terms of two concepts: personal competence and environmental press. Sometimes these concepts are amenable to beneficial manipulation, sometimes they are not. It will be useful for caregivers and healthcare practitioners to be aware of this, and to be able to tell the difference.

We suggest three applications of the P-E Model. The first is education and training of persons directly or indirectly involved in eldercare. This includes physicians, nurses and other health care professionals, social and human services workers, gerontology students and, of course, elders and their caregivers. The Model should be implemented to help explain and facilitate understanding of why particular eldercare efforts are or are not having the intended effects, and will point to options for increasing the odds of successful eldercare. The fact that it is a relatively simple, two-dimensional model should make it easily understood, even by persons with lower levels of formal education and/or whose daily lives involve mostly concrete (as distinct from conceptual) thinking.

The second application, to some extent a consequence of the first, is human services practice. The P-E Model can be used as or added to an assessment of elders and their environment, promoting a clearer understanding of what is possible and what is not and why, and suggesting what types of practice interventions or home modifications are most likely to yield successful outcomes. Often people are unaware of the totality of home- and community-based 
services available; practitioners can ensure that families have this information. Furthermore, often people are unwilling or unable to access services for which they qualify; practitioners can learn effective strategies for creating more positive attitudes toward services, and for removing any access problems.

The final area of application is at the macro, policy level. In almost any nation, older persons are a highly heterogeneous population in terms of such factors as education, income/wealth, and health/functionality. However, the lived experience of ageing in this diverse population occurs within a given political system and, typically, one dominant culture. Thus, current and future policies and programmes targeting older persons can be evaluated, using the P-E Model, as to the degree to which those policies and programmes realistically recognise and effectively promote an optimal balance between environmental press and personal competence at levels ranging from individual/familial to societal/cultural.

We believe that nations with relatively different cultures, such as Thailand, Portugal, and the United States, can beneficially apply the P-E Model to help address the issues of rapidly ageing populations. More empirical studies involving other nations will create a broader knowledge base. At this time, however, we suggest that the Person-Environment model can help families, health care professionals, and policymakers more effectively address family and societal issues of ageing.

\section{Acknowledgements}

The authors would like to acknowledge Rebecca Yenney and Caroline Landrum for their assistance.

\section{References}

Administration on Aging (AOA) (2000) Older Women (Fact Sheet) Available at: www.aoa. gov/naic/may2000/factsheets/olderwomen. html. [Date of access: 1.16.09].

Arno, P.S. (2006) Prevalence, Hours and Economic Value of Family Caregiving Family Caregiver Alliance: San Francisco, CA.

Bakas, T.; Austin, J.K.; Okonkwo, K.F.; Lewis, R.R. and Chadwick, L. (2002) 'Needs, concerns, strategies, and advice of stroke caregivers the first 6 months after discharge' Journal of Neuroscience Nursing 34:242-251.

Baker, D. (1985) 'Occupational stress' Annual Review of Public Health 6:367-381.

Baltes, M.M. and Lang, F.R. (1997) 'Everyday functioning and successful aging: The impact of resources' Psychology and Aging 12:433-443.

Berg, A.; Palomaki, H.; Lehtihalmes, M.; Lonnquist, J. and Kaste, M. (2003) 'Post stroke depression: An 18-month follow-up' Stroke 34:138-143.

Chally, P.S. and Carlson, J.M. (2004) 'Spirituality, rehabilitation and aging: A literature review' Archives of Physical Medicine and Rehabilitation 85 (Suppl. 3):S60-S65.

Chayovan, N. and Knodel, J. (1996) 'A report on the survey of the welfare of elderly in Thailand' Institute of Population Studies, Chulalongkorn University: Bangkok, Thailand.

Coffey, A. and Atkinson, P. (1996) Making Sense of Qualitative Research Data: Complementary Research Strategies Sage: Thousand Oaks, CA.

Connidis, I.A. (2001) Family Ties and Aging Sage: Thousand Oaks, CA.

Cowgill, D.O. (1974) 'Ageing and modernization: A revision of the theory' in Gubrium, J.F. (ed) Late Life: Communities and Environmental Policy Thomas: Springfield, IL.

Crandall, R.C. (1980) Gerontology: A Behavioral Science Approach Random House: New York.

Dettinger, E. and Clarkberg, M. (2002) 'Informal caregiving and retirement timing among men and women: Gender and caregiving relationships in late midlife' Journal of Family Issues 23(7):857-879.

Emlet, C.A.; Crabtree, J.L. and Condon, V.A. (2007) In-home Assessment of Older Adults: An Interdisciplinary Approach, 2nd edn, ProEd: Austin, TX.

Family Caregiver Alliance (FCA) (2003) Women and Caregiving: Facts and Figures. Available at: www.caregiver.org/caregiver/jsp/ content_node.jsp?nodeid=892 [Date of access: 1.16.09].

Family Caregiver Alliance (FCA) (2009) Caregiving Available at: http://www.caregiver.org/ caregiver/jsp/content_node.jsp?nodeid $=2313$ [Date of access: 12.11.09] 
Hooyman, N.R. and Kiyak, H.A. (2008) Social Gerontology: A Multidisciplinary Perspective, 8th edn, Pearson: Boston, MA.

INE (2007) Demographic Statistics Instituto Nacional de Estatística: Lisbon.

Jullamate, P. (2008) 'The Study of Informal Rehabilitation Performed by Thai Caregivers of Elderly Stroke Patients'. Unpublished PhD dissertation, University of Porto, Porto, Portugal.

Jullamate, P.; de Azeredo, Z.; Rosenberg, E.; Paul, C. and Subgranon, R. (2007) 'Informal stroke rehabilitation: What are the main reasons of Thai caregivers?' International Journal of Rehabilitation Research 30(4):315-320.

Kotila, M.; Numminen, H.; Waltimo, O. and Kaste, M. (1998) 'Depression after stroke: Results of the FINNSTROKE study' Stroke 29:368-372.

Lawton, M.P. and Nahemow, L. (1973) 'Ecology and the ageing process' in Eisdorfer, C. and Lawton, M.P. (eds) Psychology of Adult Development and Aging American Psychological Association: Washington, DC.

Lee, S.; Colditz, G.; Berkman, L. and Kawachi, I. (2003) 'Caregiving and risk of coronary heart disease in U.S. women: A prospective study' American Journal of Preventive Medicine 24(2):113-119.

Li, L. (2004) 'Caregiving network compositions and use of supportive services by communitydwelling dependent elders' Journal of Gerontological Social Work 43(2/3):147-164.

Link, G. and Dize, V. (2006) Family Caregiver Support: State Facts at a Glance. Available at: ncsl.org/programs/health/forum/ caregiversupport.htm [Date of access: 1.17.09].

Loeber, R. and Stouthamer-Loeber, M. (1998) 'Juvenile aggression at home and at school' in Elliot, D.S.; Hamburg, B.A. and Williams, K.R. (eds) Violence in American Schools Cambridge University Press: New York, pp. 94-126.

Mackenzie, E.R.; Rajagopal, D.E.; Meibohm, M. and Lavizzo-Mourey, R. (2000) 'Spiritual support and psychological well-being: Older adults' perceptions of the religion and health connection' Alternative Therapy 6:37-45.

Mak, A.K.M.; Mackenzie, A. and Lui, M.H.L. (2007) 'Changing needs of Chinese family caregivers of stroke survivors' Journal of Clinical Nursing 16:971-979.
MetLife (1999) The MetLife Juggling Act Study. Available at: www.caregiving.org/data/ jugglingstudy.pdf [Date of access: 1.15.09].

National Alliance for Caregiving (1998) The Caregiving Boom: Baby Boomer Women Giving Care. Available at: www.caregiving.org/ data/archives/babyboomer.pdf [Date of access: 1.19.09].

National Statistical Office (1998) The Statistics of Elderly in Thailand PA Living: Bangkok.

Navaie-Waliser, M.; Feldman, P.; Gould, D.; Levine, C.; Kuerbis, A. and Donelan, K. (2002) 'When the caregiver needs care: The plight of vulnerable caregivers' American Journal of Public Health 92(3):409-413.

Neal, A. (2007) 'Elder care: Aging in place' Saturday Evening Post 279(4):56.

Neufeld, J.E.; Rasmussen, H.N.; Lopez, S.J.; Ryder, J.A.; Magyar-Moe, J.L.; Ford, A.I.; Edwards, L.M. and Bouwkamp, J.C. (2006) 'The engagement model of person-environment interaction' Counseling Psychologist 34(2):245-259.

Older Women's League (OWL) (2003) Women and Long-term Care. Available at: www.owlnational.org [Date of access: 1.17.09].

Portnow, J. and Houtmann, M. (1987) Homecare for the Elderly: A Complete Guide McGrawHill: New York.

Pynoos, J.; Nishita, C. and Perelman, L. (2003) 'Advancements in the home modification field: A tribute to M. Powell Lawton' Journal of Housing for the Elderly 17(1/2): 105-116.

Rosenberg, E. and Gouge, N. (2007) 'Powerful tools for caregivers: Teaching skills that reduce stress and increase self-confidence' Age in Action 22(2): 1-5.

White, M.A. and Johnstone, A.S. (2000) 'Recovery from stroke: Does rehabilitation counselling have a role to play?' Disability and Rehabilitation 22:140-143.

Williams, C.L.; Rittman, M.R.; Boylstein, C.; Faircloth, C. and Haijing, Q. (2005) 'Qualitative and quantitative measurement of depression in veterans recovering from stroke' Journal of Rehabilitation Research and Development 42:277-290.

World Life Expectancy Map. Available at: www. worldlifeexpectancy.com [Date of access: 3.1.09]. 
Copyright of Health Sociology Review is the property of eContent Management Pty. Ltd. and its content may not be copied or emailed to multiple sites or posted to a listserv without the copyright holder's express written permission. However, users may print, download, or email articles for individual use. 